\title{
An evaluation of toxigenic Clostridioides difficile positivity as a patient outcome metric of antimicrobial stewardship in Saudi Arabia
}

Christopher A. Okeahialam1*, Ali A. Rabaan2 Albert Bolhuis3

1. Infection Prevention and Control, Fraser Health Authority, Surrey, BC Canada

2. Molecular Diagnostic Laboratory, Johns Hopkins Aramco Healthcare, Dhahran 31311, Saudi Arabia

3. Department of Pharmacy \& Pharmacology, University of Bath, United Kingdom

${ }^{*}$ Corresponding author*:

Christopher A. Okeahialam

Email address: jani2014.ja@gmail.com

Tel: $+1-604-360-8714$

\section{Abstract}

Background: Antimicrobial stewardship has been associated with a reduction in the incidence of health care associated Clostridium difficile infection (HA-CDI). However, CDI remains under-recognized in many low and middle-income countries where clinical and surveillance resources required to identify HA-CDI are often lacking. The rate of toxigenic C. difficile stool positivity in the stool of hospitalized patients may offer an alternative metric for these settings, but its utlity remains largely untested. 
Aim/Objective: To examine the impact of an antimicrobial stewardship on the rate of

toxigenic $\mathrm{C}$. difficile positivity among hospitalized patients presenting with diarrhoea

Methods: A 12-year retrospective review of laboratory data was conducted to compare the rates of toxigenic C. difficile in diarrhoea stool of patients in a hospital in Saudi Arabia, before and after implementation of an antimicrobial stewardship program

Result: There was a significant decline in the rate of toxigenic $C$ difficile positivity from 9.8 to $7.4 \%$ following the implementation of the antimicrobial stewardship program, and a reversal of a rising trend.

Discussion: The rate of toxigenic $\mathrm{C}$. difficile positivity may be a useful patient outcome metric for evaluating the long term impact of antimicrobial stewardship on CDI, especially in settings with limited surveillance resources. The accuracy of this metric is however dependent on the avoidance of arbitrary repeated testing of a patient for cure, and testing only unformed or diarrhoea stool specimens. Further studies are required within and beyond Saudi Arabia to examine the utility of this metric.

\section{Background}

Clostridioides difficile infection (CDI) is caused by toxigenic strains of $\mathrm{C}$. difficile (tCd), a Gram-positive, spore forming, antibiotic-resistant anaerobic bacillus. CDI is considered to be the most common cause of diarrhoea in healthcare settings in Europe and North America, and an urgent antimicrobial resistance threat (CDC, 2019). 
Most of what is known of the impact of antimicrobial stewardship on CDI has emerged from studies in North America and Europe. Systematic reviews indicate that the incidence of healthcare-associated CDI (HA-CDI) is the metric most often utilized in these studies (Feazel et al 2014, Baur et al 2017).

Other studies (Talpaert et al 2011, Jump et al 2012, Lawes et al 2017) have measured the rate of tCd positivity in diarrhoea stool as a proxy for CDI, and observed a reduction following the implementation of antimicrobial stewardship interventions. This is a simpler metric than the incidence of HA-CDI because it does not require clinical diagnosis of CDI, and does not distinguish between hospital and community-acquired CDI. It may therefore find broader applicability in settings lacking the level of surveillance and diagnostic capacity required to identify HA-CDI. A potential drawback however is that the rate of tCd positivity in a hospital setting may be inflated by a high level of asymptomatic tCd carriers in the catchment population, thereby obscuring the true impact of hospital-based stewardship interventions.

In Saudi Arabia, the few available CDI studies (Al-Tawfiq and Abed 2010, Alzaharani and Al Johani 2013, Hudhaiah and Elhadi 2019) suggest that the prevalence in Saudi communities may be under-estimated, due to a combination of low diagnostic suspicion and resources. This assertion is supported by Saudi studies on self-medication (nonprescription use) with antibiotics (Al-Mohamadi et al 2013, Alshammari et al 2017, Bin Nafisah et al 2017, Alhomoud et al 2018) that highlight unregulated and inappropriate population exposure to antibiotics, an important risk factor for $\mathrm{tCd} / \mathrm{CDI}$. 
The true burden of tCd in Saudi communities remains unknown however, and the extent to which this limits the utility of tCd positivity as a metric of hospital antimicrobial stewardship is uncertain. Given these uncertainties, we decided to compare the period positivity rate of tCd in diarrhoea stool obtained from in-patients before and after the implementation of a hospital antimicrobial stewardship program in Saudi Arabia.

\section{METHODS}

\section{Setting}

Johns Hopkins Aramco Healthcare (JHAH) is a 5-site healthcare facility in the Eastern province of Saudi Arabia. The study setting, Dhahran health centre (DHC) is the largest hospital of JHAH. Services include general medicine and surgery, intensive care, and management of haematological and solid organ malignancies. The hospital serves a catchment population of approximately 370,000 patients.

\section{Study Design}

This was retrospective study covering a twelve-year period. The study compared the rate of tCd positivity among in-patients at $\mathrm{JHAH}$, in the six year intervals before and after implementation of the antimicrobial stewardship program (ASP). The study was approved by the Institutional Research Board of JHAH.

The ASP was formally launched in 2011 , starting with education sessions specifically targeted at prescribers and hospital pharmacists (Table 1). This was preceded in 2010 however, by the introduction of restricted laboratory reporting of antibiotic 
Table 1 JHAH Antimicrobial Stewardship Interventions

\begin{tabular}{|l|l|}
\hline Intervention & Date of Implementation \\
\hline Prescriber and Pharmacist Education sessions & 2011 \\
\hline Automatic Renal Dosing & 2012 \\
\hline Antibiotic De-escalation protocol & 2012 \\
\hline Intravenous to Oral Conversion Program; & 2012 \\
\hline Vancomycin Pharmacokinetic monitoring & 2012 \\
\hline Peril-operative antibiotic protocols using adapted orders & 2012 \\
\hline $\begin{array}{l}\text { Multiple interventions including prospective audits, and } \\
\text { feedback to prescribers in paediatric outpatient clinics to } \\
\text { improve the use of antibiotics for upper respiratory tract } \\
\text { infections }\end{array}$ & 2012 \\
\hline
\end{tabular}

susceptibilities for selected Gram negative organisms (Al-Tawfiq et al, 2015). Other program components were introduced in 2012. These were automatic renal dosing, an antibiotic de-escalation protocol, intravenous to oral conversion program, vancomycin pharmacokinetic monitoring, pre-operative antibiotic protocol/adapted orders and multiple interventions including prospective audit and feedback paediatric outpatient clinics to improve prescribing practices for upper respiratory tract infections in paediatric 
outpatient clinics (Al-Tawfiq and Alawami 2017). The antimicrobial stewardship program was led by an infection disease physician and pharmacist.

\section{Outcome}

The rates of tCd positivity before and after the implementation of the ASP were estimated by dividing the number of tCd positive patients by the total number of patients tested in each period. Duplicate positive tests were excluded for each patient, except when a second positive test was obtained more than 8 weeks after the first, in which case it was considered a new infection and included in the positivity estimate.

The age group categories used were elderly (65 years and above), adult (18 to 64 years) and paediatric (3-17 years). Paediatric patients below the age of 3 were excluded from the study due to the propensity of the $0-2$ age group for asymptomatic colonization with toxigenic and non-toxigenic C. difficile strains (American Academy of Paediatrics, 2013, Antonara and Leber 2016)

\section{Microbiology}

The C. difficile policy in the study setting is to test only loose or watery stool (stool conforming to the shape of the container). Criteria for sending diarrhea or loose stool specimens to the laboratory for C.difficile testing are: 3 or more loose/watery bowel movements in a 24 hour period, bowel movements unusual or different for the patient, 
no other recognized aetiology for the diarrhea (e.g. laxative use, inflammatory bowel disease).

Over the study period, stool specimens were tested for $C$. difficile toxins $A$ and B using Enzyme-linked immunosorbent assay (Premier Toxins A\&B EIA; Meridian Bioscience). Test results and anonymised demographic data ( patients' age, gender, and in-patient location at the time of specimen collection) were received from the laboratory.

Definitions

A case of CDI was defined as a patient with a positive tCd test. Recurrent CDI (relapse) was defined as patient positive for tCd 2-8 weeks after the initial positive test.

Re-infection was defined as patient positive for tCd more than 8 weeks after the initial positive test (CDC/McDonald et al 2007, Cohen et al 2010).

Age-group-specific tCd positivity was obtained by expressing the number of tCd positive patients in a specific age group as a proportion of the number of patients tested in that group.

Statistical Analysis

Data was analysed using SPSS version 23 (SPSS Inc., Chicago, IL) for Windows. The Fisher exact test was used to test for differences in proportions. The Student t-test was used to compare the means of continuous variables. Two-sided $P$ values less than 0.05 were considered statistically significant. 


\section{RESULTS}

Over the study period (2005 to 2016), a total of 3086 inpatients at Johns Hopkins Aramco hospital (JHAH) were tested for tCd. 260 patients tested positive for tCd, giving an overall period positivity rate of $8.4 \%$.

The mean age of the study population and tCd positive patients was 55 and 59 respectively. The majority (143/260; 55\%) of tCd positive patients were elderly (65 years and over), although this age group comprised just over $40 \%$ of patients tested for tCd over the study period

A total of $12 / 260$ patient were identified with a repeat $t C d$ positive test more than 8 weeks after the first positive test. In keeping with the study case definition (CDC/McDonald et al 2007), these were considered new cases of CDI (re-infections) and included in the period positivity estimate. The majority of re-infections (8/12;66\%) were observed among the elderly. A total of $28 / 260$ patients were identified with tCd $2-8$ weeks after the initial positive test. These were considered relapses rather than new cases of CDI and excluded from the positivity estimate. The majority $(18 / 28 ; 64 \%)$ of relapses were observed in the elderly.

The upward trend in tCd positivity observed in the six-year period prior to the implementation of antimicrobial stewardship was reversed in the six-year period following the implementation of the program (figure 1). Overall, there was a significant decline $(p=0.022)$ in tCd positivity from $9.8 \%(129 / 1318)$ to $7.4 \%(131 / 1768)$ pre and post ASP respectively. 


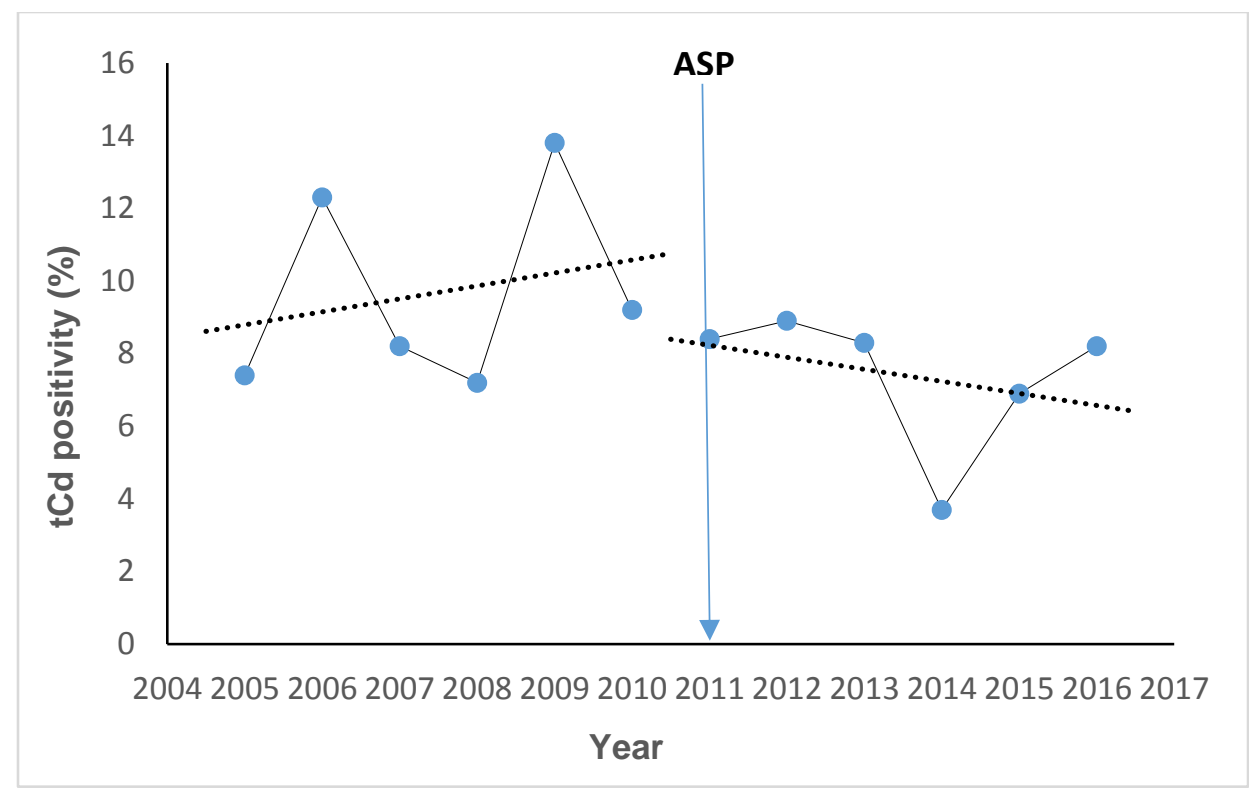

Figure 1. Secular trend in tCd positivity pre and post ASP

The decline in tCd positivity was observed in all three age groups, but this was statistically significant $(p<0.05)$ only among paediatric patients (table 2 and figure 2$)$.

Table 2 Age group-specific tCd positivity pre and post ASP

\begin{tabular}{|l|l|l|l|l|l|l|l|}
\hline Age group & $\begin{array}{l}\text { number } \\
\text { tested pre } \\
\text { ASP }\end{array}$ & $\begin{array}{l}\text { number } \\
\text { positive } \\
\text { pre ASP }\end{array}$ & $\begin{array}{l}\text { Positivity } \\
(\%) \text { pre } \\
\text { ASP }\end{array}$ & $\begin{array}{l}\text { number } \\
\text { tested } \\
\text { post ASP }\end{array}$ & $\begin{array}{l}\text { number } \\
\text { positive } \\
\text { post ASP }\end{array}$ & $\begin{array}{l}\text { Postivity } \\
(\%) \text { post } \\
\text { ASP }\end{array}$ & $P$-value \\
\hline Elderly & 548 & 64 & 11.7 & 775 & 79 & 10.2 & .419 \\
\hline Adult & 613 & 48 & 7.8 & 821 & 48 & 5.8 & .084 \\
\hline Paediatric & 157 & 17 & 10.8 & 172 & 4 & 2.3 & .002 \\
\hline Total & 1318 & 129 & 9.8 & 1768 & 131 & 7.4 & .022 \\
\hline
\end{tabular}

Pre and Post ASP decline was significant $(\mathrm{P}<.05)$ among paediatric patients 


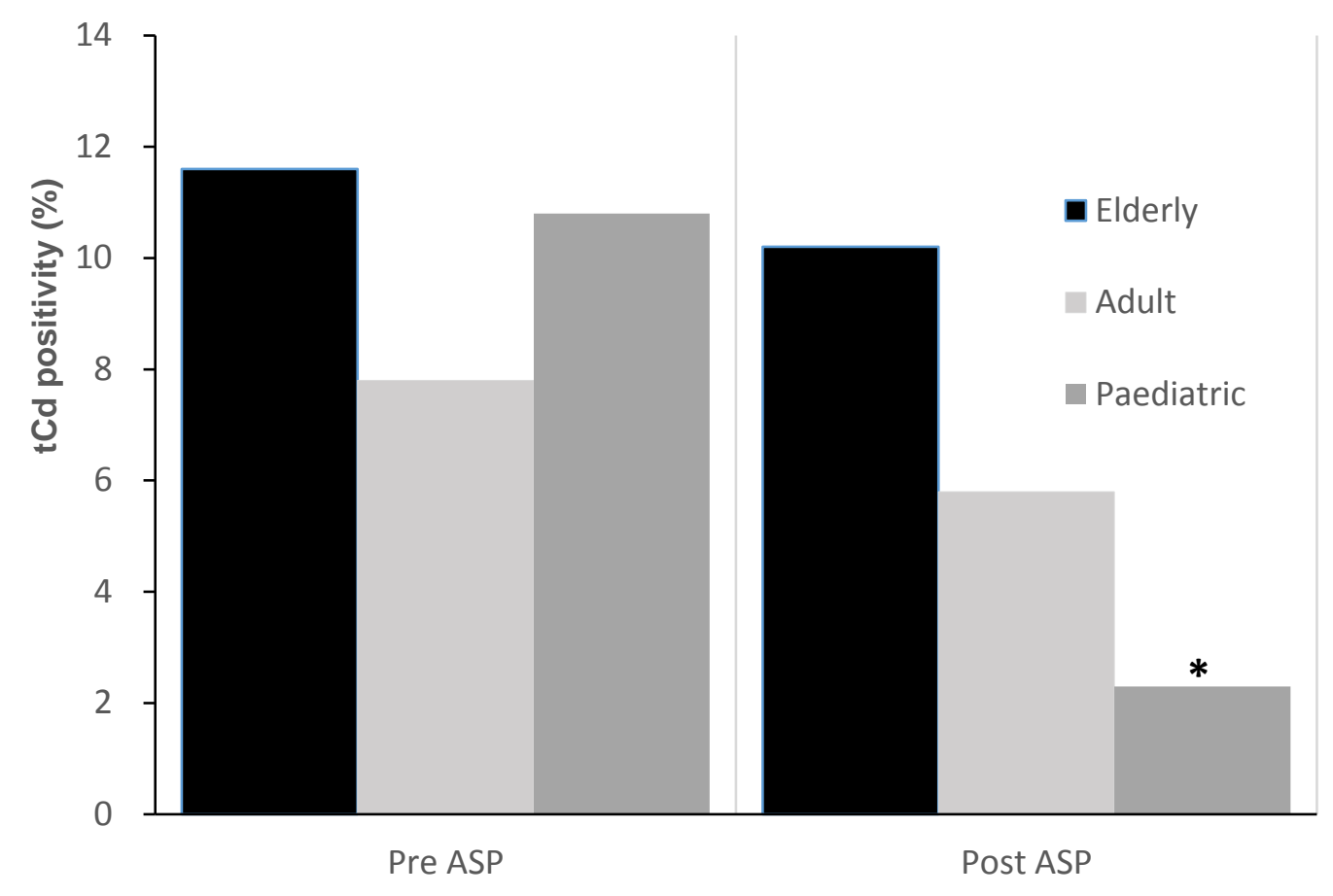

Figure 2. Age group-specific tCd positivity pre and post ASP

*Significant decline $(p<0.05)$

There were no significant differences in tCd positivity by gender, with females accounting for $139 / 260(53 \%)$ and males 121/260 (47\%) of the tCd positive patients identified.

\section{DISCUSSION}

Unlike North America and Europe from where the majority of studies on CDI epidemiology have emerged, Saudi Arabia has a predominantly young population ( $<65$ years), with the elderly ( $\geq 65$ years) accounting for less than $5 \%$ of the population (United Nations, World Population Prospects, 2019) . Therefore the predominance of the younger age groups among patients tested for C.diffiicle in the current study is consistent with this 
demographic distribution. The age-group distribution of tCd positive patients observed in the current study is also consistent with North American and European studies (McDonald et al 2006, Khanna et al 2012, Pechal et al 2016) that identify the elderly as being at especially high risk for CDI.

Although the post-ASP decline in tCd positivity was observed in all three age groups, this was statistically significant only among paediatric patients. The reason for this outcome is uncertain, but may be related to the excess burden among older patients of other CDI risk factors, including clinical co-morbidities and exposure to proton pump inhibitors and histamine 2 (h2) antagonists (Kwok et al2012, Rotman and Bishop 2013, Seto et al2014) The outcome may also reflect a higher level of compliance with antimicrobial stewardship recommendations among paediatricians, although there is no evidence in the current study to support this.

In a systematic review, Feazel et al (2014) observed that studies reporting the most impact of antimicrobial stewardship on CDI were those conducted in elderly settings, and pre-authorization of antibiotics and formulary restriction a part of the program. Therefore, a plausible explanation for the non-significant impact of the ASP on tCd positivity among the elderly in the current study is that this high-risk group was not specifically targeted for intervention. Furthermore, the ASP consisted primarily of persuasive interventions, and did not involve restriction of antibiotics. This meant that prescribers, largely, maintained autonomy and were not obliged to comply with stewardship recommendations.

The impact of the ASP on tCd positivity among elderly and adult patients may also have been dampened by the outbreak of MERS coV that occurred at the study setting in 2013, 
during the post-ASP period. MERS coV disproportionately affected these age groups (AITawfiq et al 2014), and was associated with an increase in empiric and prophylactic antibiotic use (Momattin et al 2018). This may have contributed to the reversal in 2014 of the downward trend in tCd observed in the post-ASP period (figure 2)

Despite these drawbacks, the ASP was associated with a significant overall decline in tCd positivity. Perhaps, support of the program by an infectious disease (ID) physician played a vital role in influencing antibiotic prescribing practices, and contributed to the observed effect. This assertion is consistent with other CDI studies (Jump et al 2012, Tedeschi et al2017, Ostrowsky et al 2018) highlighting the impact of ID physician support in antimicrobial stewardship, either alone, or in combination with other interventions. .

The retrospective, before-and-after intervention study design limits the extent to which the observed outcome can be attributed solely to the ASP. The influence of other related interventions implemented before the ASP, that were on going over the study period cannot be discounted. These include the hand hygiene program, and the promotion of infection prevention and environmental services best practices for CDI. It is noteworthy however, that these interventions were in place prior to the ASP, and the rising trend in tCd period positivity was reversed only after the implementation of the program in 2011.

Life expectancy has increased in Saudi Arabia, and a more than 3-fold increase in the proportion of the elderly is projected over the next two decades (United Nations, World Population Prospects 2019). With this demographic shift, the prevalence of CDI is likely to increase. To mitigate this, there is a need for concerted efforts to implement antimicrobial stewardship in health care settings, and regulate population access to 
antibiotics. Periodic measurements of in-patient tCd positivity may offer a useful means of monitoring the impact of hospital antimicrobial stewardship programs, especially in those settings lacking the resources to accurately measure the rate of HA-CDI. The accuracy of this metric is however reliant on consistent best practices among clinicians and laboratory personnel. These include the avoidance of arbitrary repeated testing of a patient for cure, and testing only unformed or diarrhoea stool specimens. This will improve efficiency, reduce bias, and enhance the predictive value of a positive tCd test. Further studies are required within and beyond Saudi Arabia to examine the utility of this metric.

\section{DECLARATIONS}

Financial support: No financial support was provided relevant to this article.

Potential conflicts of interest: All authors report no conflicts of interest relevant to this article.

\section{REFERENCES}

Alhomoud F, Aljameaa Z and Basalelaha L (2018) "Antibiotics kill things very quickly" consumers' perspectives on non-prescribed antibiotic use in Saudi Arabia BMC Public Health (18):1177 https://doi.org/10.1186/s12889-018-6088-z

Al-Mohamadi A, Badr A, Bin Mahfouz L, Samargandi D and Al Ahdal A (2013) Dispensing medications without prescription at Saudi community pharmacy: Extent and Extent and perception Saudi Pharmaceutical Journal (21): 13-18 
Alshammari TM, Alhindi SA, Alrashdi AM, Benmerzouga I and Aljofan M (2017) Pharmacy Malpractice: The rate and positivity of dispensing high-risk prescription-only medications at community pharmacies in Saudi Arabia Saudi Pharmaceutical Journal (25): 709-714

Al-Tawfiq JA and Abed M (2010) Clostridium difficile-associated disease among Patients in Dhahran, Saudi Arabia Travel Medicine and Infectious Disease (8): 373-376

Al-Tawfiq JA, Hinedi K, Ghandour J, Khairalla H, Musleh S, Ujayli A and Memish ZA (2014) Middle East Respiratory Syndrome Coronavirus: A Case-Control Study of Hospitalized Patients Clinical Infectious Diseases 59 (2):160-5

Al-Tawfiq JA, Momattin H, Al-Habboubi F and Dancer SJ (2015) Restrictive reporting of selected antimicrobial susceptibilities influences clinical prescribing Journal of Infection and Public Health (8): 234-41

Al-Tawfiq JA and Alawami AH (2017) A multifaceted approach to decrease inappropriate antibiotic use in a paediatric outpatient clinic Annals of Thoracic Medicine $12(1): 51-54$

Alzahrani N and Al Johani S (2013) Emergence of a highly resistant Clostridium difficile strain (NAP/BI/027) in a tertiary care center in Saudi Arabia Annals of Saudi Medicine 33( 2):198-199

American Academy of Pediatrics (2013) Clostridium difficile Infection in Infants and Children Pediatrics (131)196-200

Antonara S. and Leber A.L (2016) Diagnosis of Clostridium difficile Infections in Children Journal of Clinical Microbiology (54):6

Bin Nafisah S, Bin Nafesa S,.Alamery AH, Alhumaid MA, AIMuhaidib HM and Al-Eidan FA (2017) Over-the-counter antibiotics in Saudi Arabia, an urgent call for policymakers Journal of Infection and Public Health (10): 522-526 
Baur D, Gladstone BP, Burkert F, Carrara E, Foschi F, Döbele S and Tacconelli E (2017) Effect of antibiotic stewardship on the incidence of infection and colonisation with antibiotic-resistant bacteria and Clostridium difficile infection: a systematic review and meta-analysis Lancet Infectious Diseases (17) 990-1001

CDC (2019) Antibiotic Resistance Threats in the United States https://www.cdc.gov/drugresistance/pdf/threats-report/2019-ar-threats-report-508.pdf

Cohen SH, Gerding DN, Johnson S, Kelly CP, Loo VG, McDonald LC, Pepin J and Wilcox MH (2010) Clinical Practice Guidelines for Clostridium difficile Infection in Adults: 2010 Update by the Society for Healthcare Epidemiology of America (SHEA) and the Infectious Diseases Society of America (IDSA) Infection Control and Hospital Epidemiology 31 (5):431-455

Feazel LM, Malhotra A, Perencevich EN, Kaboli P, Diekema DJ and Schweizer ML (2014) Effect of antibiotic stewardship programmes on Clostridium difficile incidence: a systematic review and meta-analysis Journal of Antimicrobial Chemotherapy (69) 17481754

Hudhaiah D and Elhadi N (2019). Positivity and Genotypes of Nosocomial Clostridium difficile Infections in the Eastern Province of the Kingdom of Saudi Arabia: A MultiCentre Prospective Study Journal of Clinical and Diagnostic Research 13 (3): 16-20

Jump RLP, Olds DM, Seifi N, Kypriotakis G, Jury LA., Peron EP, Hirsch AA, Drawz PE, Watts B, Bonomo RA and Donskey C.J (2012) Effective Antimicrobial Stewardship in a Long-Term Care Facility through an Infectious Disease Consultation Service: Keeping a LID on Antibiotic Use Infection Control and Hospital Epidemiology_33 (2):1185-1192. doi:10.1086/668429

Khanna S, Pardi DS, Aronson SL, Kammer PP, Orenstein R, Sauver JL, Harmsen WS and Zinsmeister AR (2012) The epidemiology of community acquired Clostridium difficile infection: a population-based study American Journal of Gastroenterology (107):89-95 
Kwok CS, Arthur AK, Anibueze CI, Singh S, Cavallazzi R and Loke YK (2012) Risk of Clostridium difficile Infection With Acid Suppressing Drugs and Antibiotics: MetaAnalysis American Journal of Gastroenterology (107):1011-1019

Lawes T, Lopez-Lozano J, Nebot CA, Macartney G, Subbarao-Sharma R, Wares KD, Sinclair C and Gould IM (2017) Effect of a national 4C antibiotic stewardship intervention on the clinical and molecular epidemiology of Clostridium difficile infections in a region of Scotland: a non-linear time-series analysis Lancet Infectious Diseases: $(17): 194-206$

McDonald LC, Owings M and Jernigan DB (2006) Clostridium difficile infection in patients discharged from US short-stay hospitals, 1996-2003. Emerging Infectious Diseases (12):409-15.

McDonald LC, Coignard B, Dubberke E, Song X, Horan T and Kutty PK (2007) Recommendations for surveillance of Clostridium difficile-associated disease Infection Control and Hospital Epidemiology (28):140-145

Momattin H, Al-Ali AY, Mohammed K and Al-Tawfiq JA (2018). Benchmarking of antibiotic usage: An adjustment to reflect antibiotic stewardship program outcome in a hospital in Saudi Arabia Journal of Infection and Public Health (11): 310-13

Ostrowsky B, Banerjee R, Bonomo RA, Cosgrove SE, Davidson L, Doron S, Gilbert DN, Jezek A, Lynch JB, Septimus EJ, Siddiqui J and lovine NM (2018) Infectious Diseases Physicians: Leading the Way in Antimicrobial Stewardship Clinical Infectious Diseases $66(7): 995-1003$

Pechal A, Lin K, Allen S and Reveles K (2016) National age group trends in Clostridium difficile infection incidence and health outcomes in United States Community Hospitals BMC Infectious Diseases (16): 682 DOI 10.1186/s12879-016-2027-8

Rotman SR and Bishop TF (2013) Proton Pump Inhibitor Use in the U.S. Ambulatory Setting, 2002-2009 PLoS ONE 8 (2): e56060. doi:10.1371/journal.pone.0056060 
Seto CT, Jeraldo P, Orenstein R, Chia N and DiBaise J.K (2014) Prolonged use of a proton pump inhibitor reduces microbial diversity: implications for Clostridium difficile susceptibility Microbiome (2): 42

Talpaert MJ, Rao GG, Cooper BS and Wade P (2011) Impact of guidelines and enhanced antibiotic stewardship on reducing broad-spectrum antibiotic usage and its effect on incidence of Clostridium difficile infection Antimicrobial Chemotherapy (66): 2168-2174

Tedeschi S, Trapani F, Giannella M, Cristini F,Tumietto F, Bartoletti M, Liverani A, Pignanelli S, Toni L, Pederzini R, Cavina A and Viale P (2017) An Antimicrobial Stewardship Program Based on Systematic Infectious Disease Consultation in a Rehabilitation Facility Infection Control and Hospital Epidemiology 38 (1):76-82

United Nations, Department of Economic and Social Affairs, Population Divsion World Population Prospects 2019 https://population.un.org/wpp/ 